\title{
A pesquisa em linguagem e tecnologia na Universidade Federal de Minas Gerais
}

\section{Research on language and technology at Federal University of Minas Gerais}

\section{La investigación sobre lenguaje y tecnología en la Universidade Federal de Minas Gerais}

Vera Lúcia Menezes de Oliveira e Paiva, doutora em Linguística e Filologia pela Universidade Federal do Rio de Janeiro. Endereço: Rua Maria Fortunata Rotheia, 381, Condomínio Fazenda da SerraJardim Paquetá. CEP: 31330-642 - Belo Horizonte, MG. Telefones: (31) 3471-5360/ 9123-8796. E-mail: vlmop@veramenezes.com.

\section{Resumo}

Este artigo apresenta uma visão geral da pesquisa sobre linguagem e tecnologia nos últimos 12 anos na Universidade Federal de Minas Gerais, incluindo uma breve descrição da pesquisa sobre literatura e tecnologia. As teses e dissertações produzidas no Programa de Pós-Graduação em Estudos Linguísticos foram divididas em cinco grupos temáticos: características da linguagem/discurso; leitura; escrita; ensino e aprendizagem; e ambientes virtuais de aprendizagem. A grande maioria desses estudos é constituída de estudos de caso. Os dados foram coletados por meio de entrevista, observação e questionários. Há uma predominância de estudos sobre o ensino e a aprendizagem e poucos estudos sobre a interação na web. Conclui-se que a pesquisa é incapaz de acompanhar a velocidade da inovação tecnológica.

Palavras-chave: Linguagem e Tecnologia. Ensino e Aprendizagem. Leitura. Escrita. Ambientes Virtuais de Aprendizagem. Metodologia. 


\section{Abstract}

This article presents an overview of research on language and technology in the last 12 years at Universidade Federal de Minas Gerais and includes a brief description of research on literature and technology. The thesis and dissertations produced at the Graduate Program on Linguistic Studies were divided into five thematic groups: language/ discourse characteristics; reading; writing; teaching and learning; and virtual learning spaces. The great majority of these studies were case studies, and data were collected by means of observation, interview and questionnaires. There is a predominance of studies focusing on teaching and learning, but there are few studies about interaction on the web. This study reveals that research is unable to follow the speed of technology innovation.

Keywords: Language and Technology. Teaching and Learning. Reading. Writing. Virtual Learning Spaces. Methodology.

\section{Resumen}

En este artículo se presenta una visión general de la investigación sobre el lenguaje y la tecnología en los últimos 12 años en la Universidade Federal de Minas Gerais, incluyendo también una breve descripción sobre la investigación en literatura y tecnología. Las tesis y disertaciones producidas en el Programa de Posgrado en Estudios Lingüísticos se dividieron en cinco grupos temáticos: características del lenguaje / discurso, la lectura, la escritura, la enseñanza y el aprendizaje, y los entornos virtuales de aprendizaje. La gran mayoría de estos estudios consiste en estudios de caso. Se recolectaron los datos a través de entrevistas, observación y cuestionarios. Prevalecen los estudios sobre la enseñanza y el aprendizaje y hay pocos estudios sobre la interacción en la web. Se concluye que la investigación no es capaz de acompañar la velocidad de la innovación tecnológica.

Palabras clave: Lenguaje y Tecnología. Enseñanza y Aprendizaje. Lectura. Escritura. Entornos Virtuales de Aprendizaje. Metodología. 


\section{Introdução}

Os primeiros computadores chegaram ao extinto departamento de Línguas Anglo-Germânicas da Faculdade de Letras da UFMG no final da década de 80, e o primeiro laboratório, equipado com 29 computadores com acesso à Internet, entrou em funcionamento no primeiro semestre de 1997, financiado pelo Programa de Apoio à Integração Graduação/ Pós-Graduação (Proin) da Capes. Junto com os computadores, chegaram a Internet e a curiosidade sobre a mediação dessa tecnologia nas práticas de leitura e escrita, no ensino de línguas e na interação mediada por computador.

O Laboratório Proin proporcionou novas experiências pedagógicas e as primeiras pesquisas sobre linguagem e tecnologia. Paiva (1999), por exemplo, após experiências de ensino de inglês no laboratório, defendia que a introdução de uma nova metodologia de ensino mediada pelo computador podia minimizar a interferência dos fatores sociais e afetivos.

Acreditando que recursos da Internet, como o correio eletrônico e os programas de bate-papo (chats ou IRC) podem diminuir o filtro afetivo, pois os alunos interagem de uma forma mais protegida, criamos disciplinas para o desenvolvimento de leitura e escrita em inglês através da Internet. Apesar de o curso ser presencial (o professor reúne-se com os alunos no laboratório duas vezes por semana durante uma hora e 40 minutos), a comunicação acontece quase toda através de e-mail. Os alunos interagem com o professor, com os colegas e com pessoas em outros países (Ibidem, p. 367-8).

O Laboratório Proin atingiu seu objetivo de integrar ensino de graduação e a pós-graduação com o primeiro curso de língua inglesa mediado por computador, ministrado no primeiro semestre de 1997 no Laboratório Proin, que deu origem a uma investigação conduzida por um monitor da pós-graduação (SILVA, 1999). Silva fez uma avaliação do ensino e da aprendizagem de inglês por meio da Internet, com dados coletados durante as atividades de uma disciplina de aprendizagem de inglês mediada por computador.

A Faculdade de Letras extinguiu seus departamentos em 2000 e passou a se organizar de forma mais flexível em núcleos de estudos. 


\section{Dissertações e teses em linguagem e tecnologia}

Quanto à produção de dissertações e teses, os primeiros trabalhos antes da Internet focavam a leitura na tela com destaque para a produção de inferências, com suporte de metodologia experimental, como nos trabalhos de mestrado de Cláudio Gottschalg Duque - Leitura em ambiente multimídia: a produção de inferências por parte do leitor a partir da compreensão de hipertextos -, orientado por Fábio Alves e defendido em 1998, e doutorado de Carla Coscarelli - Leitura em ambiente multimídia e a produção de inferências -, orientado por Marco Antônio Vieira, em 1999².

A primeira dissertação de mestrado com foco na Internet, intitulada Novas perspectivas no ensino/aprendizagem de Francês Lingua Estrangeira, foi defendida por Fonseca em 1999, sob a orientação de Carlos Alberto Gohn. Esse trabalho, na perspectiva qualitativa, foi o primeiro de uma série de outros defendendo a pertinência do uso da Internet no ensino de línguas estrangeiras.

0 interesse pelo ensino mediado pelo computador emergia em diferentes unidades da UFMG no final dos anos 90 e início do novo século. Como exemplos, cito as dissertações de mestrado de Mônica Martins Andrade, no mestrado em Arquitetura, intitulada Computadores \& Ensino de Arquitetura, orientada por Joel Campolina, em 1999; e a de David Mattos Andrade Ávila, no mestrado de Engenharia Elétrica, intitulada Um servidor de avaliação para suporte ao ensino a distância, orientada por Luciana Enrico, em 2000.

Em 2001, com o apoio do Programa de Pós-Graduação em Estudos Linguísticos (Poslin), Paiva (2001) organizou um dos primeiros livros no Brasil sobre aprendizagem e interação mediada por computador, reunindo trabalhos de doutorandos e mestrandos do Poslin. O interesse pelo fenômeno da era digital gerou e continua gerando uma série de investigações. Há, por exemplo, várias pesquisas sobre o fenômeno da Internet no Programa de Pós-Graduação em Educação.

2 Na verdade, esses

dois trabalhos tiveram como orientador inicial o professor Mike Dillinger.
Ao pesquisar na Biblioteca Digital de teses e dissertações da UFMG as pesquisas defendidas no Programa de Pós-Graduação em Educação, encontramos trabalhos que também investigam a questão da 
linguagem, especialmente, o letramento digital, mas optei, neste artigo, por concentrar a pesquisa nos trabalhos produzidos na Faculdade de Letras.

A seguir, passo à análise da pesquisa produzida na Faculdade de Letras, envolvendo os seus dois programas: um em Estudos Linguísticos e outro em Estudos Literários. Começamos pelo último, pois ele tem pouca produção associada à tecnologia. Apesar de o livro e o cinema serem também tipos de tecnologia, darei prioridade aos trabalhos associados ao uso da Internet e farei rápidas menções a trabalhos envolvendo o rádio e a televisão.

\section{Literatura e tecnologia}

Os Estudos Literários apresentam apenas quatro trabalhos com foco em literatura e tecnologia digital, sendo três dissertações de mestrado e uma tese de doutorado. Há ainda poucos trabalhos sobre rádio e televisão e muitas teses e dissertações relacionadas ao cinema.

A primeira dissertação de Rena (2006), intitulada Do autor tradicional ao Agenciador Cibernético: do biopoder à biopotência, discute a nova figura do autor enquanto produtor na rede telemática. A segunda, de Pereira (2007), intitula-se No Limite da Ficção: comparações entre Literatura e RPG - Role Playing Games. Nesse trabalho, o autor discute "a negação do status de ficção de um modo de narrar não consagrado pela sociedade" (p. 2) nos Role Playing Games(RPG) ou jogos de interpretação de papéis.

Os outros dois trabalhos têm a poesia como objeto de estudo. Aguiar (2008), em sua dissertação, analisa os poemas-processo e a videopoesia de Elson Froes, disponíveis na página do poeta na Internet e que apresentam, segundo o pesquisador, características visuais, verbográficos e de animação. Garcia (2008), na tese A literatura como design gráfico: da poesia concreta ao poema-processo de Wlademir Dias Pino, investiga as relações entre a literatura e o design gráfico, estudando o poema-processo nas obras de Wlademir Dias Pino, no contexto da computação gráfica e das mídias digitais. 
A maioria da pesquisa em linguagem e tecnologia se concentra no programa em Estudos Linguísticos.

\section{Linguística, linguística aplicada e tecnologia}

A linha de pesquisa em Linguagem e Tecnologia no Programa de Pós-Graduação em Estudos Linguísticos, na Universidade Federal de Minas Gerais, foi criada em 2007, mas a pesquisa em torno do tema se iniciou na década de 90, como vimos na introdução deste texto.

Mesmo após a criação da linha, alguns trabalhos associados à linguagem e tecnologia continuam sendo defendidos em outras linhas do programa, o que demonstra que as tecnologias de informação e a comunicação atraem os pesquisadores de diversas vertentes na área da linguagem. A maioria dos trabalhos investiga questões associadas à Internet, mas há também alguns trabalhos sobre rádio, jornal e televisão. A tabela, a seguir, indica o número de teses e dissertações a partir do ano 2000. Os dados de 2012 são referentes ao primeiro semestre apenas.

Tabela 1. Dissertações e teses defendidas nos últimos 12 anos

\begin{tabular}{|c|c|c|}
\hline Ano & Dissertações & Teses \\
\hline 2000 & 1 & - \\
\hline 2001 & - & - \\
\hline 2002 & 1 & 1 \\
\hline 2003 & 4 & 1 \\
\hline 2004 & 5 & - \\
\hline 2005 & 2 & 1 \\
\hline 2006 & 1 & - \\
\hline 2007 & 5 & 1 \\
\hline 2008 & 5 & 6 \\
\hline 2009 & 3 & 1 \\
\hline 2010 & 3 & 1 \\
\hline 2011 & 5 & 3 \\
\hline 2012 & 1 & 3 \\
\hline Total & 36 & 18 \\
\hline
\end{tabular}


Foram produzidas, de 2000 até junho de 2012, 54 pesquisas de mestrado e de doutorado. Das 36 dissertações defendidas nos últimos 11 anos e meio, temos duas sobre rádio (uma em 2005 e outra em 2007); três sobre televisão (uma em 2007 e duas em 2009); e uma sobre leitura de gráficos no jornal impresso. Dentre as 18 teses, há duas sobre televisão e uma sobre vídeo.

A seguir, apresento uma análise dos temas e da metodologia das pesquisas com foco nas tecnologias da Internet.

\section{a. Os temas}

A pesquisa nos últimos 11 anos e meio se distribui nos seguintes eixos temáticos: características da linguagem/discurso; leitura; escrita; ensino e aprendizagem; e ambientes virtuais de aprendizagem.

\section{a.1. Características da linguagem/discurso}

A primeira pesquisa (SOUZA, 2000), nessa perspectiva, investiga as características do chat. Posteriormente, Neto (2011) também estudou características desse gênero, detendo-se no estudo da forma "Aqui". Neto conclui que essa forma "é responsável por demarcar a introdução ou fechamento de um novo tópico discursivo, mantendo a coerência textual” (p. 7) no gênero bate-papo, como registrado em seu resumo.

Pereira (2002) estudou os reparos em interações em fórum on-line e Fleischer (2003) pesquisou padrões de comportamento de um fórum em termos da participação e colaboração de seus membros. Ferreira (2006) fez uma comparação da linguagem de blogs de adolescentes e de adultos à luz da teoria semiolinguística; e Cohen (2007) também fez um estudo comparativo, estudando anúncios publicitários veiculados em grandes portais da Internet e revistas impressas, sob a ótica da análise do discurso.

Vieira (2009) estudou a referenciação metalinguística nas interações mediadas pelo computador e comparou as interações mediadas pelo computador com as interações face a face e a interação mediada pelo texto impresso. 
Outro gênero estudado foi a webpage. Frade (2012) fez uma análise dos aspectos multimodais e de design na estrutura do gênero website educacional dirigido a crianças de seis a 10 anos de idade.

A única rede social que mereceu um estudo em termos de linguagem foi o Orkut, na tese de Carvalho (2012), que investigou, na perspectiva semiótica, as configurações sintáticas e semânticas no agenciamento do conteúdo da amizade.

\section{a.2. Leitura}

Com a chegada da Internet, uma curiosidade que gerou muitas pesquisas foi se o texto na tela mudava o processo de leitura. A comparação entre leitura no papel e na tela foi o tema das pesquisas de mestrado e de doutorado de Ribeiro (2003 e 2008). Novais (2008) estudou a leitura das interfaces gráficas do computador e concluiu que esse tipo de leitura é diferente de outras práticas letradas.

Outro tema que tem sido alvo de pesquisas é o hipertexto. Araújo (2003) defendeu que toda leitura é um processo hipertextual, e Caetano (2004), também investigando a leitura hipertextual, concluiu, como registra seu resumo, "que links encontrados na cadeia anafórica do texto apresentam melhores possibilidades de construção de sentido do que links aleatórios” (p. 7). Dias (2008), por meio de experimentos, avaliou a compreensão de leitores expostos a diferentes formas de organização da página inicial e do menu de navegação de um hipertexto. Já Gualberto (2008) estudou a influência dos hiperlinks na leitura de hipertexto enciclopédico digital.

\section{a.3. Escrita}

A inovação tecnológica trouxe novas formas de escrever acompanhadas de suspeitas e preconceitos. A crença de que a forma abreviada de escrita nos chats pode influenciar negativamente nas produções de outros gêneros por adolescentes, por exemplo, é negada na dissertação de Santos (2003). 
O fenômeno da Wikipédia também levanta curiosidade entre os pesquisadores, e duas pesquisas, uma de mestrado (SERRANO, 2011) e outra de doutorado (D' ANDREA, 2011), tiveram como tema essa enciclopédia virtual colaborativa. Serrano (2011) examinou a dinâmica da prática editorial da Wikipédia, sob a ótica da teoria semiótica greimasiana; e D'Andrea analisou os processos auto-organizados e colaborativos durante a atualização de uma amostra de biografias de pessoas vivas nessa enciclopédia colaborativa virtual.

\section{a.4. Ensino e aprendizagem}

O ensino e a aprendizagem mediados por computador é um tema que atrai grande interesse de mestrandos e doutorandos. Três pesquisas sobre o ensino mediado por computador tiveram como foco a percepção de alunos (ALMEIDA, 2004) e de professores (CORRÊA, 2002; LACERDA, 2012) sobre o uso da Internet como auxiliar no ensino de línguas.

Colaboração e estratégias de aprendizagem também foram temas investigados. Uma tese (SOUZA, 2003) e uma dissertação (BRAGA, 2004) investigaram a telecolaboração entre aprendizes de português no exterior trabalhando em pares com aprendizes de inglês no Brasil. Souza (2003) priorizou as questões da autonomia e da colaboração observadas em um projeto teletandem entre aprendizes de português na Austrália e de inglês no Brasil. Braga (2004) investigou autonomia e estratégias de aprendizagem (sociais e de compensação) utilizadas por aprendizes americanos e brasileiros aprendendo, respectivamente, português e inglês, também em um projeto teletandem.

Estratégias de aprendizagem e autonomia foram também investigadas por Sabariz (2004) com dados coletados em um curso de inglês de leitura e escrita on-line, ministrado na Faculdade de Letras da UFMG. Veado (2008), em um estudo de caso, pesquisou a colaboração na produção textual em ambiente virtual com uma ferramenta wiki e Bohn (2010), também em um estudo de caso, observou como os aprendizes utilizaram recursos da web 2.0 em aulas de língua inglesa em uma disciplina da graduação em Letras na UFMG. 
Duas pesquisas tiveram como objeto de estudo os jogos eletrônicos. Santos (2011) investigou, em sua dissertação, como os jogos MMORPG auxiliam no processo de aquisição, e Teixeira (2011) estudou o processo de pesquisa escolar na web de alunos das séries finais do ensino fundamental, habituados a navegar na web. Almeida (2011), em sua tese de doutorado, verificou a validade ecológica de um jogo simulador de voo para PC como ferramenta de suporte para o estudo do uso situado de inglês como L2 por pilotos.

Outra preocupação presente em alguns trabalhos diz respeito à apropriação e à difusão da tecnologia pelas escolas. Pordeus (2004) registrou em seu resumo que, naquela época, havia razões pelas quais a utilização da tecnologia ainda era restrita: "falta de estrutura nas escolas, falta de computador por parte dos alunos, falta de tempo e falta de capacitação do professor" (p. 12). Oito anos depois, Teixeira (2012) conclui que os computadores chegaram às escolas, mas, como apontam alguns professores, eles ainda não se sentem à vontade com a inovação tecnológica. Já Almeida (2007) investigou o processo de implementação de atividades de CALL nas aulas de línguas de uma instituição de ensino privado, e Silva (2008a) acompanhou processo semelhante em uma sala de aula de uma escola pública.

Outro tipo de apropriação é tratado por Faria (2010). Ela estudou a apropriação do Orkut como ambiente virtual de aprendizagem e defendeu que o uso desse tipo de rede social constitui importante fator de motivação da aprendizagem.

O discurso do professor sobre sua própria prática no ambiente virtual também é objeto de investigação. O trabalho de Pereira (2010), sob a ótica da semiótica, investiga o discurso de 13 professoras de disciplinas introdutórias de língua portuguesa a distância no âmbito do ensino superior.

\section{a.5. Ambientes virtuais de aprendizagem}

A sala de aula virtual é outro grande tema nos estudos sobre linguagem e tecnologia. Nos estudos realizados no Poslin, prevalecem 
investigações à luz dos sistemas complexos. No primeiro trabalho nessa perspectiva, Parreira (2005) estudou duas salas de aula digital com alunos de pós-graduação. O estudo teve por objetivo compreender a dinâmica das interações e as possíveis implicações pedagógicas que fatores como feedback e design podem ter no processo de ensino e aprendizagem e no gerenciamento de cursos mediados pelas tecnologias de informação e comunicação.

Braga (2007) investigou uma sala de aula virtual de alunos da graduação bastante peculiar em uma disciplina de Linguística Aplicada ofertada pela Faculdade de Letras da UFMG, durante o primeiro semestre letivo de 2004. A disciplina era constituída por comunidades autônomas de aprendizagem on-line, isto é, uma sala de aula subdividida em subgrupos que trabalharam autonomamente de forma fractal, já que exibiam as mesmas características do grande grupo, tais como reciprocidade, construção compartilhada de significado e liderança distribuída.

Silva (2008b) pesquisou a interação e a colaboração entre alunos de uma sala de aula virtual de pós-graduandos como eventos dinâmicos, e Martins (2008a), em uma perspectiva ecológica, comparou a emergência de eventos complexos em aulas de produção de texto em inglês na graduação em Letras da UFMG com atividades on-line e face a face. Souza (2011) estudou a dinamicidade e a adaptabilidade em comunidades virtuais de aprendizagem de alunos da graduação interagindo em ambientes virtuais de aprendizagem distintos, Moodle e Teleduc. Seu estudo teve como suporte teórico, além da complexidade, a textografia de Swales (1998).

A tecnologia tem sido vista na literatura sobre ensino mediado pelo computador como importante fator para o incremento da motivação na aprendizagem de línguas. É o que demonstra, por exemplo, Oliveira (2005), em seu estudo de caso sobre tarefas comunicativas integradas ao meio virtual.

A avaliação de software educacional foi um tema raro nesses 12 anos. $O$ único registro é o trabalho de Souza (2007), que, além de avaliar 
quatro softwares diferentes para o ensino de italiano, propôs uma lista de checagem para esse tipo de avaliação. A webrádio também foi tema de estudo em apena uma tese (MARTINS, 2008b).

\section{A metodologia}

Nos trabalhos analisados, verifiquei que os métodos de pesquisa utilizados foram, em sua maioria, qualitativos, com exceção das investigações sobre leitura, que utilizaram, prioritariamente, experimentos.

A pesquisa qualitativa parece ser uma característica dominante da pesquisa brasileira quando comparada à pesquisa sobre linguagem e tecnologia nos Estados Unidos e no Japão, por exemplo. Artigos de pesquisadores desses dois países publicados em revistas internacionais como CALICO, CALL, ReCALL e IJCALLT, geralmente, relatam resultados de experimentos, utilizando grupos de pesquisa e controle e análises estatísticas dos dados.

No corpus analisado neste artigo, predominam os estudos de caso que utilizam a observação não-participante, os questionários e as entrevistas para verificar a percepção de alunos e de professores sobre o ensino de línguas mediado pelo computador. Uma questão preocupante é que muitos desses estudos de caso se dão em contextos criados especialmente para a pesquisa. Isso nos leva a levantar a hipótese de que a tecnologia da Internet não está, ainda, totalmente integrada ao ambiente escolar brasileiro, pois não há indicação de que experiência semelhante teria prosseguimento após a pesquisa.

\section{Conclusão}

A análise das pesquisas de mestrado e doutorado realizadas no Programa de Pós-Graduação em Estudos Linguísticos da UFMG revela que a maior parte das pesquisas se concentra em questões voltadas para o ensino de língua inglesa e a leitura em língua portuguesa. 
Há poucas pesquisas sobre a produção textual na Internet e sobre a interação virtual. Não há, até o momento, trabalhos de pesquisa sobre a produção de texto hipertextual, nem mesmo nos trabalhos sobre ensino. Uma hipótese para esse fenômeno pode ser o fato de os pesquisadores concentrarem sua atenção naquilo que ensinam e de não terem adotado ainda uma escrita hipertextual na escola. Não há também trabalhos investigando a produção de textos orais (ex: podcasts) ou de vídeos e quadrinhos com ferramentas digitais.

Há uma única dissertação sobre blog e não há, também, nenhuma pesquisa sobre a linguagem do twitterou do facebook. Há duas pesquisas sobre o Orkut, mas apenas uma delas investiga a natureza da linguagem.

Outras ausências observadas são a investigação sobre o uso de mensagens mediadas por telefones celulares e também sua aplicação no ensino; e o uso de aplicativos para os tablets.

A tecnologia se desenvolve com muita rapidez e a produção de pesquisa não tem conseguido acompanhar a velocidade dessas inovações tecnológicas. Uma sugestão seria fomentar estudos para investigar questões relevantes que ainda não conseguiram motivar os pesquisadores e seus mestrandos e doutorandos.

Recebido em 30/12/2012

Aprovado em 11/03/2013

\section{Referências bibliográficas}

AGUIAR, J. M. M. Elson Fróes: poesia visual na internet. 2008. Dissertação (Mestrado em Letras: Estudos Literários)- Faculdade de Letras, Universidade Federal de Minas Gerais, Belo Horizonte, 2008.

\section{ALMEIDA, D. C. de. Do quadro de giz para a tela do computador:} percepções de estudantes universitários sobre a utilização de tarefas online em um curso de inglês instrumental semipresencial. 2004. 
Dissertação (Mestrado em Estudos Linguísticos)- Faculdade de Letras, Universidade Federal de Minas Gerais, Belo Horizonte, 2004.

Validade Ecológica de um Simulador de Voo para PC no Uso do Inglês como L2. 2011. Tese (Doutorado em Estudos Linguísticos)Faculdade de Letras, Universidade Federal de Minas Gerais, Belo Horizonte, 2011.

ALMEIDA, V. C. Trilhando novos caminhos: professores rumo à implementação de CALL. 2007. Dissertação (Mestrado em Estudos Linguísticos)- Faculdade de Letras, Universidade Federal de Minas Gerais, Belo Horizonte, 2007.

ANDRADE, M. M. Computadores \& Ensino de Arquitetura. 1999. Dissertação (Mestrado em Estudos Linguísticos)- Faculdade de Arquitetura, Universidade Federal de Minas Gerais, 1999.

ARAÚJO, M. A. de. Compreensão de Hipertexto sob a Perspectiva da Teoria da Mesclagem. 2003. Dissertação (Mestrado em Estudos Linguísticos)- Faculdade de Letras, Universidade Federal de Minas Gerais, Belo Horizonte, 2003.

ÁVILA, D. M. A. Um servidor de avaliação para suporte ao ensino a distância. 2000. Dissertação (Mestrado em Engenharia Elétrica)-Escola de Engenharia, Universidade Federal de Minas Gerais, Belo Horizonte, 2000.

$\mathrm{BOHN}, \mathrm{V}$. C. R. Uso dos recursos da Web 2.0 nas aulas de língua inglesa: colaboração e produção de conhecimento na rede. 2010. Dissertação (Mestrado em Estudos Linguísticos)-Faculdade de Letras, Universidade Federal de Minas Gerais, Belo Horizonte, 2010.

BRAGA, J. de C. F. Aprendizagem de línguas em regime tandem via e-mail: colaboração, autonomia e estratégias sociais e de compensação. 2004. Dissertação (Mestrado em Estudos Linguísticos)- Faculdade de Letras, Universidade Federal de Minas Gerais, Belo Horizonte, 2004. 


\section{Comunidades autônomas de aprendizagem on-line na}

Perspectiva da Complexidade. 2007. Tese (Doutorado em Estudos Linguísticos)- Faculdade de Letras, Universidade Federal de Minas Gerais, Belo Horizonte, 2007.

CAETANO, P. L. 0 uso de links e leitura no ciberespaço: a interação leitorhipertexto e a construção de sentido. 2004. Dissertação (Mestrado em Estudos Linguísticos)- Faculdade de Letras, Universidade Federal de Minas Gerais, Belo Horizonte, 2004.

CARVALHO, W. F. de. 0 discurso da intimidade: a paixão semiótica - amizade - nas comunidades do Orkut. 2012. Tese (Doutorado em Estudos Linguísticos)- Faculdade de Letras, Universidade Federal de Minas Gerais, Belo Horizonte, 2012.

COHEN, S. A. de M. 0 discurso publicitário virtual x impresso: enunciação e contrato: uma análise comparativa. 2007. Dissertação (Mestrado em Estudos Linguísticos)- Faculdade de Letras, Universidade Federal de Minas Gerais, Belo Horizonte, 2007.

CORRÊA, E. F. A Internet como ferramenta auxiliar em sala de aula de língua inglesa: percepções de duas professoras. 2002. Dissertação (Mestrado em Estudos Linguísticos)- Faculdade de Letras, Universidade Federal de Minas Gerais, Belo Horizonte, 2002.

D' ANDREA, C. F. de B. Processos editoriais auto-organizados na Wikipédia em português: a edição colaborativa de "Biografias de Pessoas Vivas”. 2011. Tese (Doutorado em Estudos Linguísticos)- Faculdade de Letras, Universidade Federal de Minas Gerais, Belo Horizonte, 2011.

DIAS, M. C. A influência do modo de organização de hipertextos na compreensão. 2008. Dissertação (Mestrado em Estudos Linguísticos)Faculdade de Letras, Universidade Federal de Minas Gerais, Belo Horizonte, 2008.

DUQUE, C. G. Leitura em ambiente multimídia: a produção de inferências por parte do leitor a partir da compreensão de hipertextos. 1998. 
Dissertação (Mestrado em Estudos Linguísticos)- Faculdade de Letras, Universidade Federal de Minas Gerais, Belo Horizonte, 1998.

FARIA, H. de O. Socializando e Aprendendo: a incorporação da sociedade virtual Orkut ao ensino de língua inglesa. 2010. Dissertação (Mestrado em Estudos Linguísticos)- Faculdade de Letras, Universidade Federal de Minas Gerais, Belo Horizonte, 2010.

FERREIRA, F. B. Comunicação virtual: uma análise contrastiva da linguagem de blogs de adolescentes e de adultos à luz da teoria semiolinguística. 2006. Dissertação (Mestrado em Estudos Linguísticos)Faculdade de Letras, Universidade Federal de Minas Gerais, Belo Horizonte, 2006.

FLEISCHER, E. A colaboração online como subsídio para o desenvolvimento profissional de professores de línguas. 2003. Dissertação (Mestrado em Estudos Linguísticos)- Faculdade de Letras, Universidade Federal de Minas Gerais, Belo Horizonte, 2003.

FONSECA, L. R. de C. e. Internet: Novas perspectivas no ensino/ aprendizagem de Francês Língua Estrangeira. 1999. Dissertação (Mestrado em Estudos Linguísticos)-Faculdade de Letras, Universidade Federal de Minas Gerais, Belo Horizonte, 1999.

FRADE, S. M. Análise dos aspectos multimodais e de design na estrutura de websites educacionais: inglês para crianças de 6 a 10 anos. 2012. Dissertação (Mestrado em Estudos Linguísticos)- Faculdade de Letras, Universidade Federal de Minas Gerais, Belo Horizonte, 2012.

GARCIA, A. M. A literatura como design gráfico: da poesia concreta ao poema-processo de Wlademir Dias Pino. 2008. Tese (Doutorado em Letras: Estudos Literários)- Faculdade de Letras, Universidade Federal de Minas Gerais, Belo Horizonte, 2008.

GUALBERTO, I. M. T. Influência dos hiperlinks na leitura de hipertexto enciclopédico digital. 2008. Tese (Doutorado em Estudos Linguísticos)Faculdade de Letras, Universidade Federal de Minas Gerais, Belo Horizonte, 2008. 
LACERDA, N. A. Linguagem e cognição: categorização e significado das concepções de educadores sobre tecnologia digital. 2012. Tese (Doutorado em Estudos Linguísticos)- Faculdade de Letras, Universidade Federal de Minas Gerais, Belo Horizonte, 2012.

MARTINS, A. C. S. A emergência de eventos complexos em aulas on-line e face-a-face: uma abordagem ecológica. 2008. Tese (Doutorado em Estudos Linguísticos) - Faculdade de Letras, Universidade Federal de Minas Gerais, Belo Horizonte, 2008a.

MARTINS, N. P. M. Webradio: Novos gêneros, novas formas de interação. 2008. Tese (Doutorado em Estudos Linguísticos)- Faculdade de Letras, Universidade Federal de Minas Gerais, Belo Horizonte, 2008b.

NETO, E. P. Um novo olhar sobre o uso da forma lexical "aqui", no gênero textual bate-papo por computador, à luz da teoria da estrutura retórica. 2011. Dissertação (Mestrado em Estudos Linguísticos)- Faculdade de Letras, Universidade Federal de Minas Gerais, Belo Horizonte, 2011.

NOVAIS, A. E. C. Leitura nas interfaces gráficas de computador: compreendendo a gramática da interface. 2008. Dissertação (Mestrado em Estudos Linguísticos)- Faculdade de Letras, Universidade Federal de Minas Gerais, Belo Horizonte, 2008.

OLIVEIRA, S. M. G. P. de. A motivação em uma tarefa de keypal na aprendizagem de inglês: um estudo descritivo. 2005. Dissertação (Mestrado em Estudos Linguísticos)- Faculdade de Letras, Universidade Federal de Minas Gerais, Belo Horizonte, 2005.

PAIVA, V. L. M. de O. e. Diários online na aprendizagem de língua inglesa mediada por computador. In: MARI, H. et al. (Orgs.). Fundamentos e Dimensões da Análise do Discurso. Belo Horizonte: Carol Borges, 1999. p. 359-378.

(Org.). Interação e Aprendizagem em ambiente virtual. Belo Horizonte: Faculdade de Letras/UFMG, 2001. 
PARREIRA, V. A. A sala de aula digital sob a perspectiva dos sistemas complexos: uma abordagem qualitativa. 2005. Tese (Doutorado em Estudos Linguísticos)- Faculdade de Letras, Universidade Federal de Minas Gerais, Belo Horizonte, 2005.

PEREIRA, D. R. M. Atividades Didáticas para Ensino de Português em Ambiente Digital: uma análise semiótica. 2010. Dissertação (Mestrado em Estudos Linguísticos)- Faculdade de Letras, Universidade Federal de Minas Gerais, Belo Horizonte, 2010.

PEREIRA, F. E. L. No Limite da Ficção: comparações entre Literatura e RPG - Role Playing Games. 2007. Dissertação (Mestrado em Letras: Estudos Literários)- Faculdade de Letras, Universidade Federal de Minas Gerais, Belo Horizonte, 2007.

PEREIRA, J. A. M. Mecanismos de edição e auto-edição interacional: mal entendido e reparo na interação via e-mail em contexto educacional. 2002. Tese (Doutorado em Estudos Linguísticos)- Faculdade de Letras, Universidade Federal de Minas Gerais, 2002.

PORDEUS, I. A construção do ciberprofessor para o ensino de língua estrangeira: qual a distância entre teoria e prática? 2004. Dissertação (Mestrado em Estudos Linguísticos)-Faculdade de Letras, Universidade Federal de Minas Gerais, Belo Horizonte, 2004.

RENA, A. S. A. Do autor tradicional ao Agenciador Cibernético: do biopoder à biopotência. 2006. Dissertação (Mestrado em Letras: Estudos Literários)-Faculdade de Letras, Universidade Federal de Minas Gerais, Belo Horizonte, 2006.

RIBEIRO, A. E. F. Ler na Tela - Novos Suportes para Velhas Tecnologias. 2003. Dissertação (Mestrado em Estudos Linguísticos)- Faculdade de Letras, Universidade Federal de Minas Gerais, Belo Horizonte, 2003.

Navegar lendo, ler navegando: aspectos do letramento digital e da leitura de jornais. 2008. Tese (Doutorado em Estudos Linguísticos)Faculdade de Letras, Universidade Federal de Minas Gerais, Belo Horizonte, 2008. 
SABARIZ, S. A. Aprendizagem de língua inglesa via Internet: estratégias de aprendizagem e manifestações de autonomia do aprendiz. 2004. Dissertação (Mestrado em Letras: Estudos Literários)- Faculdade de Letras, Universidade Federal de Minas Gerais, Belo Horizonte, 2004.

SANTOS, E. M. dos. O Chat e sua influência na escrita do adolescente. 2003. Dissertação (Mestrado em Estudos Linguísticos)- Faculdade de Letras, Universidade Federal de Minas Gerais, Belo Horizonte, 2003.

SANTOS, V. R. F. S. Os jogos MMORPG como auxilares no processo de aquisição de língua inglesa. 2011. Dissertação (Mestrado em Estudos Linguísticos)- Faculdade de Letras, Universidade Federal de Minas Gerais, Belo Horizonte, 2011.

SERRANO, P. H. S. M. Coerência entre princípios e práticas na Wikipédia Lusófona: uma análise semiótica. 2011. Dissertação (Mestrado em Estudos Linguísticos)- Faculdade de Letras, Universidade Federal de Minas Gerais, Belo Horizonte, 2011.

SILVA, D. C. Um estudo de caso da implementação de aulas de inglês informatizadas na escola de ensino fundamental. 2008. Dissertação (Mestrado em Estudos Linguísticos)-Faculdade de Letras, Universidade Federal de Minas Gerais, Belo Horizonte, 2008a.

SILVA, R. C. Teaching and learning English through Internet. In: ENCONTRO NACIONAL E PROFESSORES UNIVERSITÁRIOS DE LÍNGUA INGLESA, 14., 1997, Belo Horizonte. Anais... Belo Horizonte: Departamento de Letras Anglo-Germânicas/UFMG, 1999. p.284-292.

SILVA, V. A dinâmica caleidoscópica do processo de aprendizagem colaborativa no contexto virtual: um estudo na perspectiva da complexidade/caos. 2008. Tese (Doutorado em Estudos Linguísticos)Faculdade de Letras, Universidade Federal de Minas Gerais, Belo Horizonte, 2008b.

SOUZA, R. F. Avaliação de Softwares para o Ensino de Línguas: foco na compreensão oral. 2007. Dissertação (Mestrado em Estudos 
Linguísticos)- Faculdade de Letras, Universidade Federal de Minas Gerais, Belo Horizonte, 2007.

SOUZA, R. A. de. $\mathbf{O}$ chat em língua inglesa: interações nas fronteiras da oralidade e da escrita. 2000. Dissertação (Mestrado em Estudos Linguísticos)- Faculdade de Letras, Universidade Federal de Minas Gerais, Belo Horizonte, 2000.

Aprendizagem de línguas em tandem: estudo da telecolaboração através da comunicação mediada pelo computador. 2003. Tese (Doutorado em Estudos Linguísticos)-Faculdade de Letras, Universidade Federal de Minas Gerais, Belo Horizonte, 2003.

SOUZA, V. V. S. Dinamicidade e adaptabilidade em comunidades virtuais de aprendizagem: uma textografia à luz do paradigma da complexidade. 2011. Tese (Doutorado em Estudos Linguísticos)- Faculdade de Letras, Universidade Federal de Minas Gerais, Belo Horizonte, 2011.

SWALES, J. Other floors, other voices: a textography of a small university building. London: Lawrence Erlbaum Associates, 1998.

TEIXEIRA, A. G. D. Difusão Tecnológica no Ensino de Línguas: o uso de computadores portáteis nas aulas de Língua Portuguesa sob a ótica da Complexidade. 2012. Tese (Doutorado em Estudos Linguísticos)Faculdade de Letras, Universidade Federal de Minas Gerais, Belo Horizonte, 2012.

TEIXEIRA, S. A. Fazendo pesquisa escolar na internet. 2011. Dissertação (Mestrado em Estudos Linguísticos)-Faculdade de Letras, Universidade Federal de Minas Gerais, Belo Horizonte, 2011.

VEADO, M. da C. M. Colaboração no processo de produção textual em uma atividade online: um estudo de caso com o gênero resenha de filme. 2008. Dissertação (Mestrado em Estudos Linguísticos)- Faculdade de Letras, Universidade Federal de Minas Gerais, Belo Horizonte, 2008. 
VIEIRA, M. S. de P. A referenciação metalinguística nas inter@ções mediadas pelo computador. 2009. Tese (Doutorado em Estudos Linguísticos)- Faculdade de Letras, Universidade Federal de Minas Gerais, Belo Horizonte, 2009. 